Edema macular diabético

\title{
Diabetic macular edema
}

\author{
J. Andonegui, L. Jiménez Lasanta
}

\section{RESUMEN}

El edema macular diabético representa la primera causa de pérdida visual en los pacientes con diabetes mellitus. Su complejidad, unida a la aparición de nuevos métodos de diagnóstico así como de novedosas alternativas de tratamiento, hace que el enfoque de esta enfermedad suponga un importante reto para el oftalmólogo. A lo largo de este artículo se describen su patofisiología, manifestaciones clínicas, clasificación, diagnóstico y tratamiento, haciendo especial énfasis en los nuevos métodos diagnósticos y en las diferentes opciones terapéuticas.

Palabras clave. Edema macular diabético. Diabetes mellitus.

An. Sist. Sanit. Navar. 2008; 31 (Supl. 3): 35-44.

\begin{abstract}
Diabetic macular edema is the principal cause of visual loss in patients with diabetes mellitus. Its complexity, together with the appearance of new methods of diagnosis and new alternatives for treatment, mean that the approach to this disease is an important challenge for ophthalmologists. This article describes its pathophysiology, clinical manifestations, classification, diagnosis and treatment, with special emphasis on the new diagnostic methods and on the different therapeutic options.
\end{abstract}

Key words. Diabetic macular edema. Diabetes mellitus.
Servicio de Oftalmología. Hospital de Navarra. Pamplona.

\section{Correspondencia:}

José Andonegui Navarro

Servicio de Oftalmología

Hospital de Navarra

Irunlarrea 3

31008 Pamplona

Tfno. 848422081

E-mail: jandonen@cfnavarra.es 


\section{INTRODUCCIÓN}

La diabetes mellitus es una entidad cuya prevalencia en la población de los países desarrollados está entre un 6 y un $8 \%$. Aunque tanto la retinopatía diabética proliferativa como el edema macular diabético pueden provocar pérdida visual, se acepta que es el edema la principal causa de pérdida visual en los pacientes afectados por esta enfermedad ${ }^{1}$.

El abordaje de este proceso presenta varios retos para el oftalmólogo. En primer lugar y en lo que se refiere al diagnóstico, está claro que los métodos clásicos para la detección de esta enfermedad, como son el examen biomicroscópico, la oftalmoscopía indirecta o la angiografía con fluoresceína (AGF), se han visto superados por nuevos métodos como la tomografía óptica de coherencia (OCT). Algunos autores incluso sugieren que los distintos tipos de edema macular detectados con la OCT podrían representar entidades clínicas diferentes que requerirían tratamientos específicos ${ }^{2}$. Pero tampoco el enfoque terapéutico del edema macular diabético está del todo claro en la actualidad. A los métodos tradicionales de control metabólico ${ }^{3,4} \mathrm{y}$ fotocoagulación con láser ${ }^{5}$ se han unido en los últimos años nuevas alternativas como la inyección intravítrea de triamcinolona ${ }^{6.8}$ o de otras sustancias antiangiogénicas ${ }^{9,10} \mathrm{o}$ el tratamiento quirúrgico mediante vitrectomía ${ }^{11,12}$. La ausencia hasta el momento de grandes estudios prospectivos randomizados para evaluar la mayor parte de estas terapias hace difícil determinar cuál es el tratamiento más adecuado en cada circunstancia.

\section{PATOFISIOLOGÍA}

La alteración histológica encontrada en el edema macular diabético es una acumulación de líquido en la capa de Henle y la capa nuclear interna de la retina. Este líquido proviene del compartimiento intravascular y su flujo, como en el resto de los tejidos, está modulado por el balance entre la presión hidrostática y la presión osmótica. Pero en la retina existe otra estructura, que es la barrera hematoretiniana (BHR), que también actúa regulando la permeabilidad vascular. Esta BHR se divide en dos partes, una interna formada por el endotelio vascular de la retina y otra externa, que la constituyen las uniones estrechas de las células del epitelio pigmentario de la retina.

El principal mecanismo que conduce al acúmulo extracelular de líquido en la retina es una alteración en la permeabilidad de la BHR. Parece ser que la hiperglucemia mantenida provoca, por mecanismos no del todo conocidos en la actualidad, un aumento en la producción de factor de crecimiento del endotelio vascular (VEGF) por parte de las células gliales, microgliales y neuronales de la retina. La hipoxia sería otro factor implicado en el aumento de la producción de VEGF ${ }^{13,14}$. El VEGF, además de ser una sustancia con capacidades angiogénicas, tiene una muy importante actividad permeabilizante y actuaría aumentando la permeabilidad de la BHR y facilitando la extravasación de líquido del compartimiento intravascular. La mayor susceptibilidad de la mácula a la formación de edema podría deberse al hecho de que esta zona de la retina posee muy pocos astrocitos. Estas células sintetizan factores que aumentarían la sintesis de proteínas de las uniones estrechas de la BHR y disminuirían por tanto su permeabilidad.

También los factores sistémicos pueden favorecer el desarrollo del edema macular diabético. La hipertensión provoca un incremento en la presión hidrostática de los capilares retinianos. Otras circunstancias como la hipoalbuminemia van a disminuir la presión oncótica. Ambas circunstancias conducen a un aumento de la permeabilidad vascular y favorecen la aparición de edema.

\section{MANIFESTACIONES CLÍNICAS}

La principal causa de pérdida visual en los pacientes diabéticos es el edema macular diabético ${ }^{1}$. La alteración que define la aparición del edema macular diabético es un engrosamiento de la retina en el área macular provocado por la extravasación de líquido al espacio extravascular. Este engrosamiento puede ser detectado en el examen biomicroscópico o por medio de dispositivos diagnósticos como la OCT. El engrosamiento de la retina puede acompañarse de exudados duros, que están for- 
mados por material lipídico y proteináceo extravasado de los vasos de la retina y depositado en las capas externas de la retina o incluso en ocasiones en el espacio subretiniano (Fig.1).

La manifestación clínica más relevante en el edema macular diabético es una disminución visual central, asociada a una deformación de las imágenes, que puede aparecer en estadios muy tempranos de la enfermedad. El edema macular diabético puede desarrollarse asociado a diferentes grados de retinopatía diabética, que pueden ir desde una retinopatía no proliferativa moderada hasta una retinopatía diabética proliferativa avanzada ${ }^{15}$. Es más frecuente en personas de edad avanzada y con diabetes tipo II.

\section{CLASIFICACIÓN}

Hasta la aparición de la OCT el edema macular diabético se clasificaba en focal o difuso. El edema focal se caracteriza por la aparición de exudados duros circinados alrededor de grupos de microaneurismas. En la AGF se evidencia un escape focal generalmente a través de microaneurismas. En el edema difuso no es tan evidente la aparición de exudados o de microaneurismas y en la AGF aparece un escape difuso en toda la zona macular ${ }^{15}$ (Fig. 2).

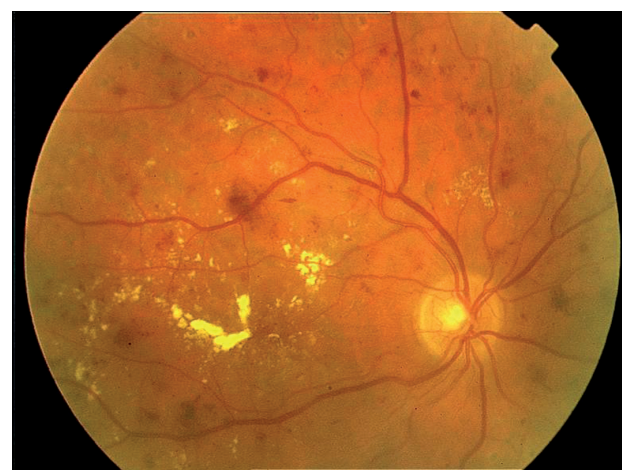

Figura 1. Exudados duros maculares en un paciente con edema macular diabético. También se pueden ver otras alteraciones propias de la retinopatía diabética como hemorragias puntiformes o en llama y microaneurismas.
El Early Treatment Diabetic Retinopathy Study (ETDRS) ${ }^{5}$ introdujo el término de edema macular diabético clínicamente significativo, que se define como:

1. Engrosamiento retiniano a 500 micras o menos del centro de la fóvea.

2. Exudados duros a 500 micras o menos del centro de la fóvea si se asocian con engrosamiento retiniano adyacente.

3. Engrosamiento retiniano de al menos un disco de área y que parte del mismo se encuentre dentro de un disco de diámetro del centro de la fóvea.

Según el ETDRS serían los pacientes cuyo edema pudiese encuadrarse como clínicamente significativo los que se beneficiarían del tratamiento con láser y de ahí la importancia de esta clasificación.

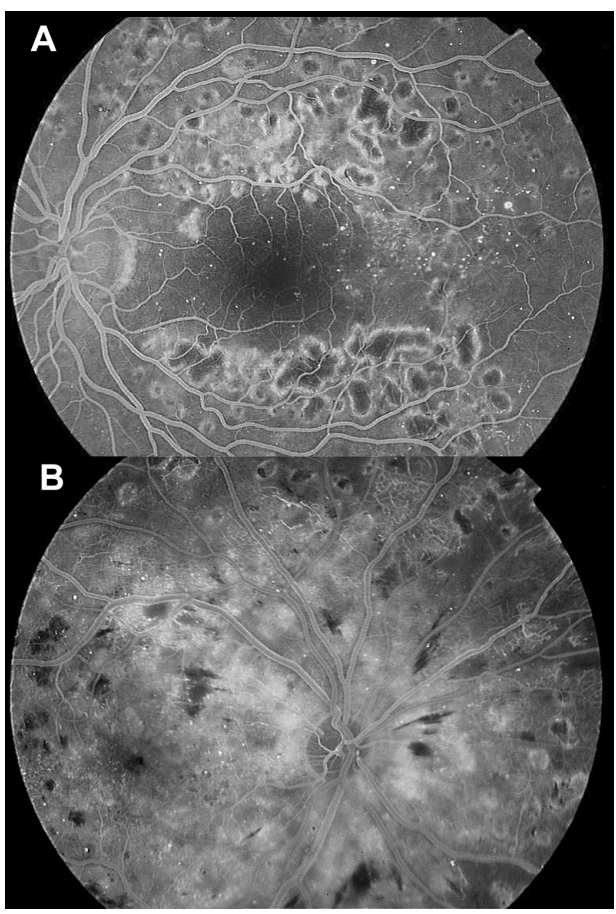

Figura 2. Tipos angiográficos de edema macular diabético. A: edema macular focal. Fuga de colorante en los microaneurismas paramaculares. B: edema difuso. Escape difuso en toda la red capilar perifoveal. 
De forma más reciente se ha propuesto la Clasificación Internacional de la Retinopatía Diabética ${ }^{16}$, que en lo que se refiere al edema macular diabético propone tres grados:

1. Leve. Engrosamiento retiniano o exudados duros en el polo posterior pero lejos del centro de la mácula.

2. Moderado. Cercanía del engrosamiento o los exudados al centro de la mácula.

3. Severo. Los exudados o el engrosamiento afectan al centro de la macula.

La introducción del OCT al diagnóstico del edema macular diabético ha hecho que se propongan nuevas clasificaciones. Otani describe tres tipos de edema basados en los cambios estructurales hallados en el $\mathrm{OCT}^{17}$. El engrosamiento espongiforme de la retina se caracteriza por un aumento moderado del grosor de la mácula y la aparición de áreas de baja reflectividad en las capas externas de la retina. El edema macular quístico muestra grandes espacios quísticos que ocupan todo el grosor de la retina y provocan una importante distorsión de la anatomía macular. El desprendimiento seroso subfoveal se manifiesta como una zona hiporeflectiva de configuración fusiforme en el espacio subfoveal. Kim propone una nueva clasificación en la que incluye los tres tipos descritos por Otani, aunque al engrosamiento espongiforme pasa a denominarlo engrosamiento difuso de la retina. Además añade otros dos tipos, que son la tracción hialoidea posterior sin desprendimiento de retina traccional y la tracción hialoidea posterior con desprendimiento de retina ${ }^{2}$ (Fig. 3 ).

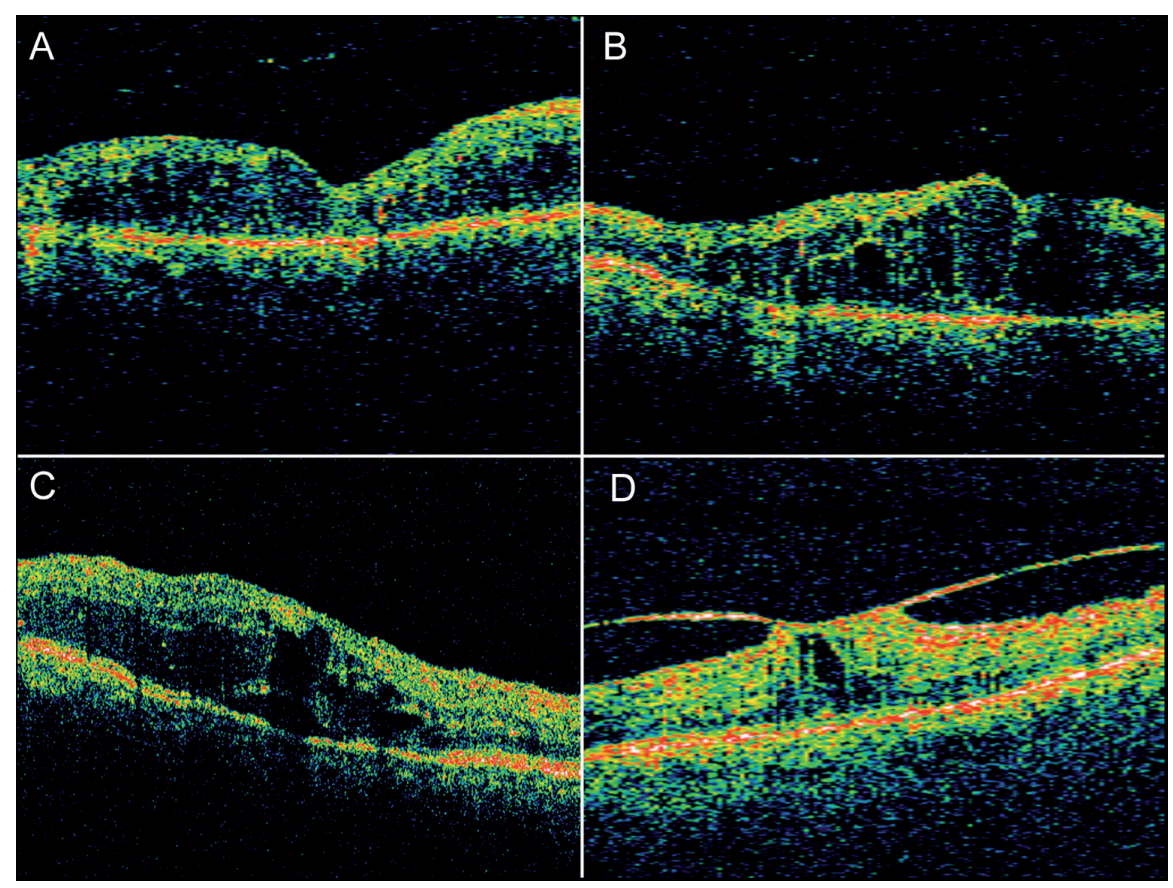

Figura 3. Tipos de edema macular diabético según la OCT. A: engrosamiento difuso de la retina. La retina está aumentada de grosor y muestra una disminución de la reflectividad en las capas externas, pero conserva su configuración anatómica con la depresión foveal central. B: edema macular quístico. Se forman grandes cavidades quísticas en todo el espesor de la retina, cuya anatomía está totalmente desestructurada. C: desprendimiento seroso subfoveal. Acúmulo de líquido seroso en el espacio subfoveal. Además en este caso existen cavidades quísticas en el resto del espesor retiniano. D: Tracción hialoidea posterior. La hialoides posterior tracciona de la mácula provocando un desprendimiento central. 


\section{DIAGNÓSTICO}

El método utilizado clásicamente para diagnosticar el edema macular diabético ha sido el examen biomicroscópico. El problema de este método es que la información que proporciona es exclusivamente cualitativa y subjetiva. Visualizar los exudados duros no representa ningún problema, pero detectar la presencia de engrosamiento de la retina puede resultar más complejo y los hallazgos encontrados pueden ser muy variables en función del observador. Además estos métodos son poco sensibles puesto que se necesitan grandes variaciones en el grosor de la retina para que puedan ser detectadas mediante el examen biomicroscópico ${ }^{18}$.

También la AGF se ha empleado para diagnosticar el edema macular diabético. La interpretación de los resultados de esta prueba es también subjetiva y aunque es útil para detectar el escape vascular, éste no siempre está asociado a engrosamiento de la retina ${ }^{5}$, que es lo que define la presencia o no de edema macular diabético. La AGF se utilizaba para establecer la división del edema macular diabético en focal o difuso pero, como veremos más adelante, resulta más útil basarnos en los datos de la OCT. La AGF tendría actualmente dos indicaciones. La primera es detectar puntos focales de fuga para guiar el tratamiento con láser. La segunda es valorar la presencia de isquemia macular, que es un factor de muy mal pronóstico en lo que a la recuperación visual se refiere.

La OCT es hoy en día la prueba más útil para diagnosticar y clasificar el edema macular diabético. Los cortes tomográficos pueden detectar de forma objetiva y con precisión la presencia de líquido en las capas de la retina y permiten rastrear la existencia de zonas de engrosamiento retiniano, que además pueden ser medidas teniendo así datos objetivos para valorar la evolución y la respuesta al tratamiento de estos pacientes (Fig. 4). También permi-
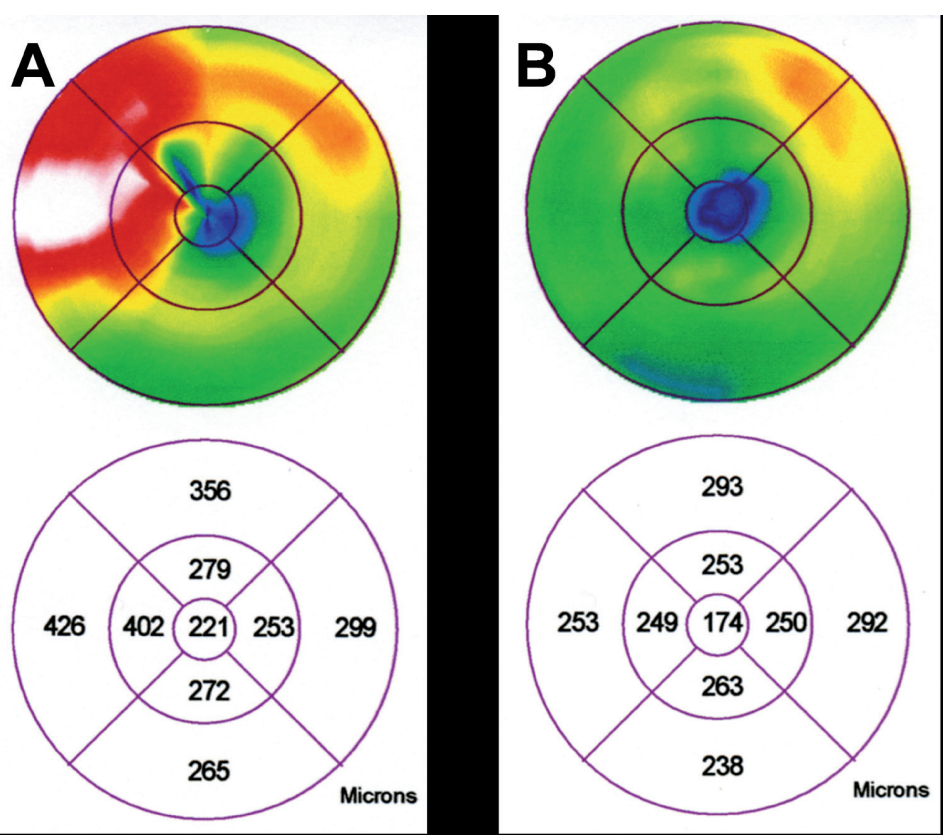

Figura 4. Medición del grosor retiniano del paciente de la figura 5 mediante OCT. A: antes del tratamiento con láser se aprecia un incremento del grosor en la parte izquierda de la imagen, que corresponde a la zona de exudación. B: después del tratamiento con láser el grosor de la retina en la zona tratada ha disminuido. 
te examinar con detalle la interfase vítreomacular y detectar posibles alteraciones susceptibles de tratamiento quirúrgico, muy frecuentes en los pacientes diabéticos. Los diversos tipos de edema macular diabético detectados mediante la OCT $^{2,17}$ podrían constituir entidades clínicas diferenciadas y tener diferentes indicaciones en cuanto al tratamiento.

\section{TRATAMIENTO}

Hasta la fecha tan solo el control metabólico $^{3,4}$ y la fotocoagulación con láser ${ }^{5}$ (Fig. 5) han demostrado de forma concluyente su eficacia en el tratamiento del edema macular diabético mediante estudios prospectivos randomizados. El ETDRS demostró que la fotocoagulación focal con láser era capaz de frenar la pérdida visual en pacientes con edema macular clínicamente significativo ${ }^{5}$. Parece ser que el efecto del láser en el edema macular diabético es, por una parte, destruir fotorreceptores en la zona macular para disminuir la hipoxia en esta zona. La hipoxia ha sido implicada en el desarrollo del edema macular diabético ${ }^{13}$, posiblemente por el aumento que provoca en la producción de $\mathrm{VEGF}^{14}$. También el láser podría actuar induciendo la proliferación de las células endoteliales y de las células del epitelio pigmentario retiniano. De esta forma, las células destruidas por el láser serían sustituidas por otras nuevas, de mejor funcionalidad, con lo que mejoraría la eficacia de la BHR interna y externa en el control del edema $^{19}$. A pesar de estos supuestos buenos resultados obtenidos en el ETDRS, menos de un $3 \%$ de estos pacientes experimentan mejoría en su visión, 12 \% continúan perdiendo agudeza visual a pesar del tratamiento y en $40 \%$ de ellos persiste el edema pasados 12 meses $^{20}$. También se ha compro-

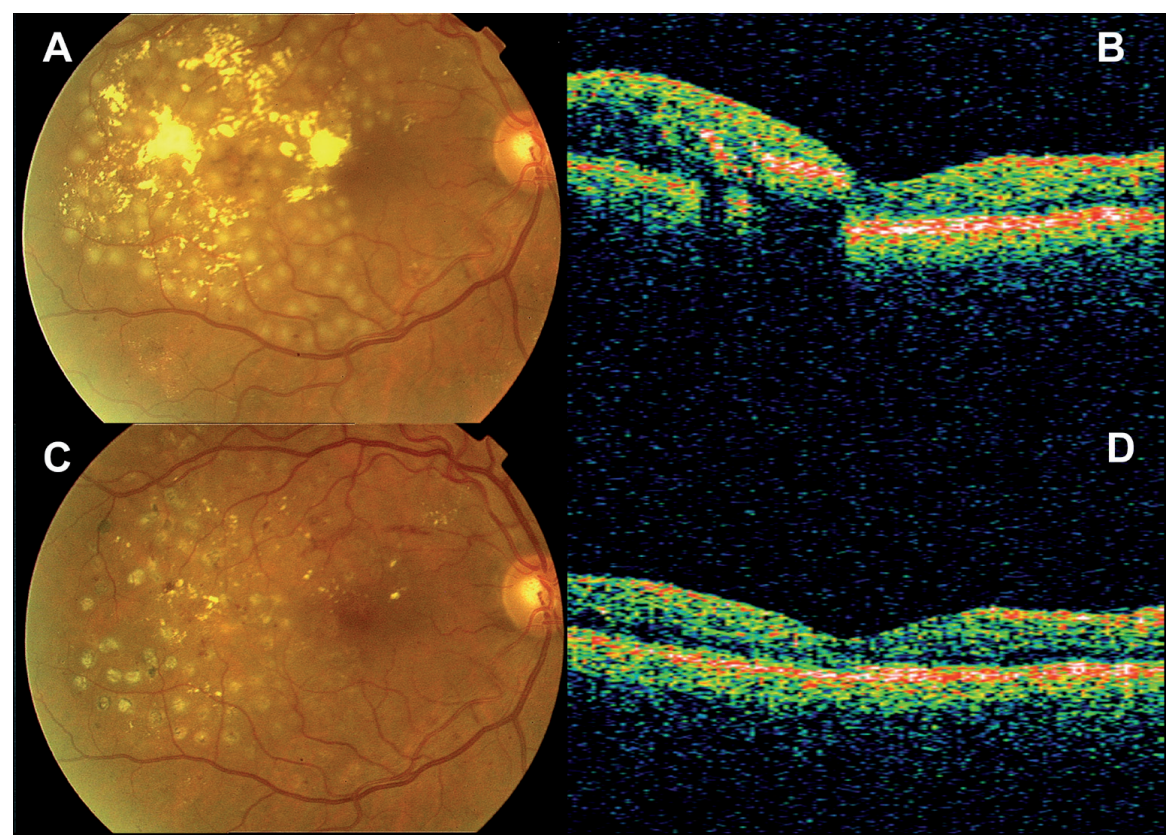

Figura 5. Tratamiento con láser en un paciente con edema macular diabético. A: Grandes exudados duros en la zona temporal de la mácula. Se aprecian los impactos de láser en rejilla en toda la zona de exudación. B: OCT del mismo paciente. Se observan los exudados duros intraretinianos y el engrosamiento difuso de la retina en la zona afectada. C: aspecto de la retina a los ocho meses del tratamiento con láser. Se evidencian las cicatrices de la fotocoagulación. Los exudados duros están en franca regresión. D: en la OCT se puede ver la desaparición de los exudados intraretinianos y la disminución del grosor de la retina tratada. 
bado que aquellos pacientes cuyo edema se clasificaba como de tipo difuso serían refractarios al tratamiento con láser ${ }^{21,22}$.

Los malos resultados visuales obtenidos con el láser y su incapacidad para tratar los edemas difusos han hecho surgir en los últimos años alternativas de tratamiento como la vitrectomía o la inyección intravítrea de triamcinolona o de otras sustancias antiangiogénicas. En lo que respecta a la vitrectomía, Lewis fue el primero en plantear que éste era un tratamiento efectivo en aquellos casos en los que el edema no había respondido al láser y se asociaba a la presencia de una hialoides posterior engrosada y tensa ${ }^{23}$. Posteriormente esta modalidad terapéutica ha sido aplicada también a pacientes cuya hialoides posterior no estaba engrosada ${ }^{24} \mathrm{e}$ incluso en casos en los que estaba despren$\operatorname{dida}^{25}$. De forma más reciente se ha introdu- cido el pelado rutinario de la membrana limitante interna (MLI) en los pacientes con edema macular diabético que eran tratados mediante vitrectomía ${ }^{26,27}$. La mayor parte de los estudios efectuados para comprobar la validez de este procedimiento incluyen un número escaso de pacientes y arrojan resultados contradictorios. Mientras que algunos encuentran mejoría tanto anatómica como funcional ${ }^{11}$, otros describen sólo mejoría en el aspecto anatómico de la mácula, pero sin cambios en la agudeza visual, sugiriendo incluso que el pelado de la MLI podría conducir a alteraciones estructurales de la retina que impedirían una adecuada recuperación visual ${ }^{12}$. Probablemente, mientras no aparezcan estudios que demuestren lo contrario, parece razonable restringir el uso de la vitrectomía en el edema macular diabético a aquellos casos en los que la OCT

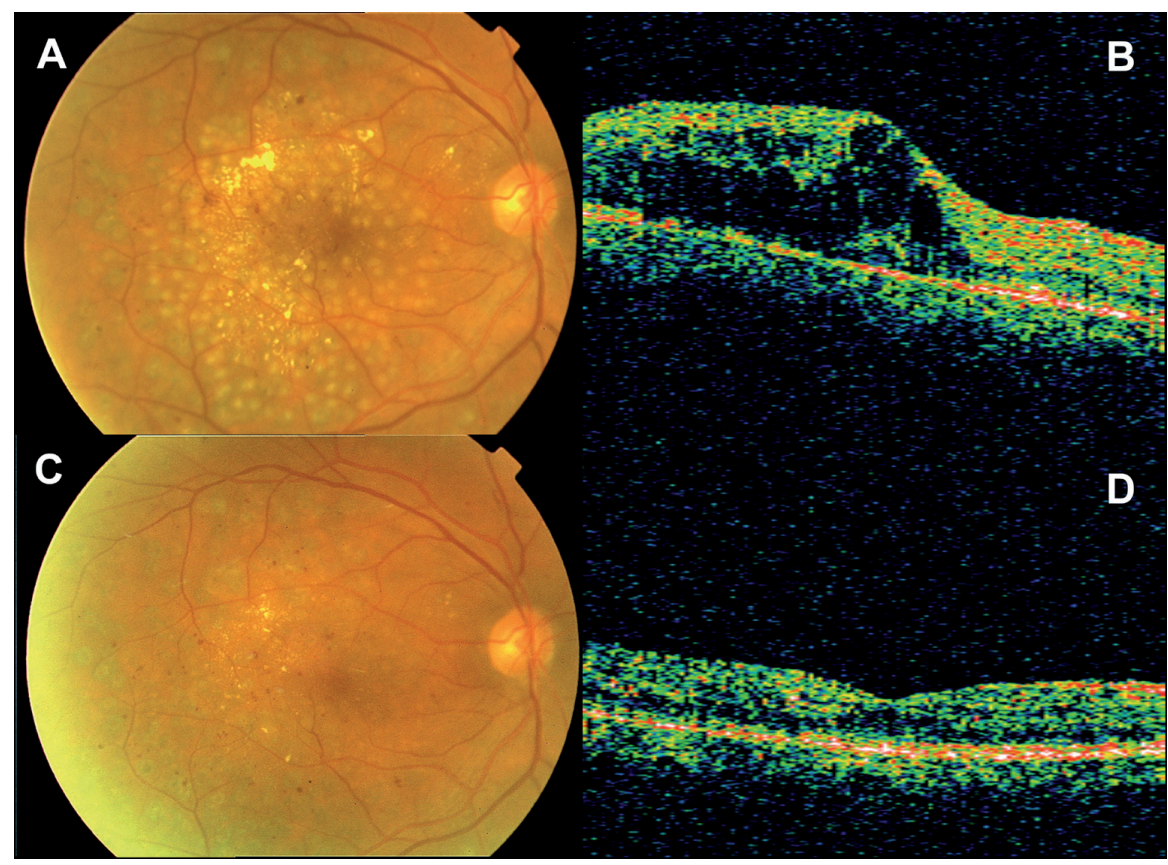

Figura 6. Tratamiento con triamcinolona intravítrea y fotocoagulación con láser en un paciente con edema macular diabético. A: En la fotografía de fondo se aprecian exudados duros maculares y los impactos de láser en rejilla en la zona macular. Un mes antes del láser a este paciente se le habían inyectado $4 \mathrm{mg}$ de triamcinolona intravítrea. B: OCT realizada antes de la inyección de triamcinolona que muestra un edema de tipo quístico y un pequeño desprendimiento seroso subfoveal. C: aspecto de la retina once meses después del tratamiento. Los exudados están en regresión y se observan unas leves cicatrices de láser. D: OCT realizada a los once meses del tratamiento en la que se aprecia una completa resolución del edema. 
demuestre tracción sobre la zona macular o importantes alteraciones de la interfase vitreoretiniana (Fig. 6).

Otro tratamiento que ha despertado gran interés y ha levantado enormes expectativas para el tratamiento del edema macular diabético es la inyección intravítrea de triamcinolona ${ }^{6-8}$. Esta sustancia actuaría reduciendo temporalmente la permeabilidad de los capilares o disminuyendo la producción de $\mathrm{VEGF}^{28}$, pero no actúa sobre la hipoxia, que podría estar implicada en el desarrollo del edema macular. Uno de los inconvenientes que presenta este tratamiento es que aunque a corto plazo se obtiene una desaparición del edema y una mejoría de la visión en un gran porcentaje de pacientes, su eficacia es temporal ${ }^{29}$ y a medio plazo la recidiva del edema es la norma ${ }^{30}$. Por otra parte, un importante porcentaje de los pacientes que reciben esta terapia desarrollan elevación de la presión intraocular o catarata. Además, recientemente han sido publicados los resultados de un estudio multicéntrico elaborado en Estados Unidos por la Red para la Investigación Clínica de la Retinopatía Diabética que demuestran que a largo plazo la triamcinolona aislada es peor que la fotocoagulación con láser para el tratamiento del edema macular diabético ${ }^{31}$.

Para intentar mejorar los pobres resultados obtenidos con la inyección aislada de triamcinolona, se ha propuesto combinar este tratamiento con la fotocoagulación con láser. Se plantea una secuencia en la cual primero se realizaría la inyección intravítrea de Triamcinolona y unas semanas después, cuando el edema hubiese desaparecido, se realizaría la fotocoagulación en rejilla en la zona macular (Fig. 7). En teoría este procedimiento permitiría aplicar el láser de forma más precisa y con menos intensidad al hacerlo sobre una

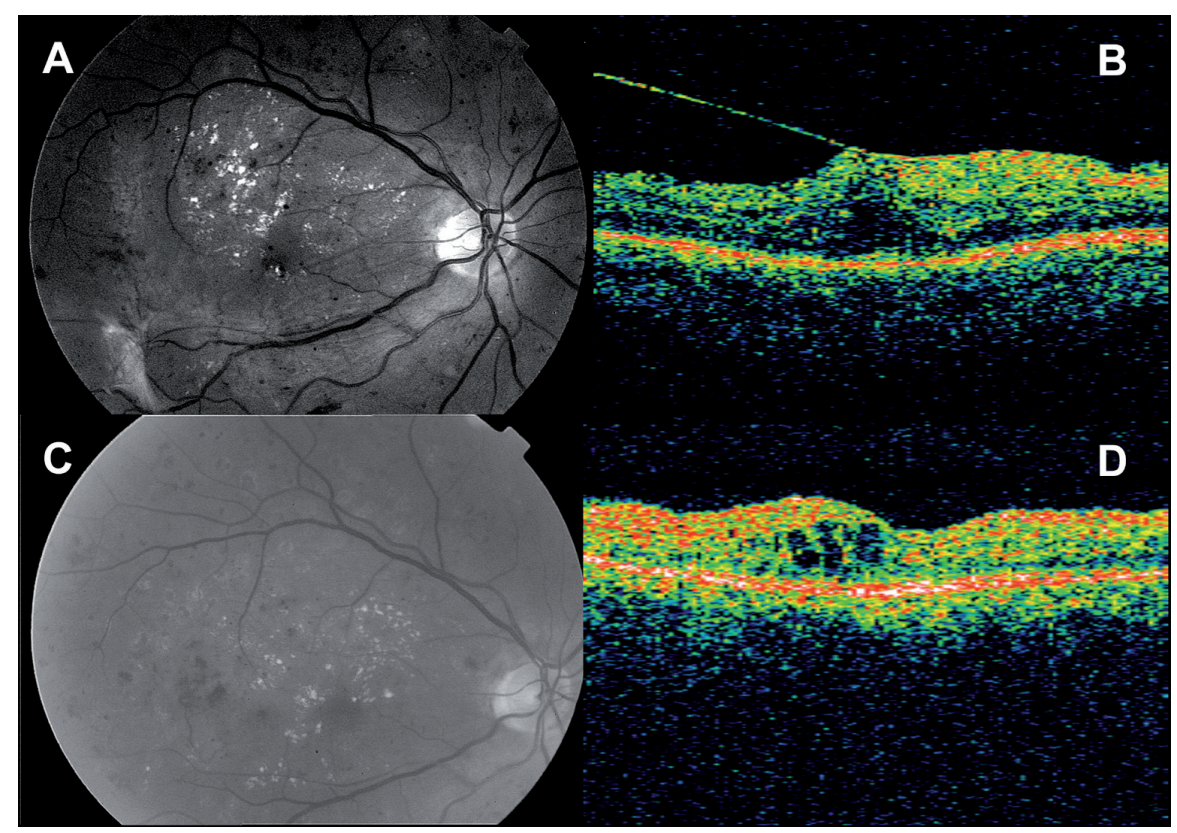

Figura 7. Tratamiento mediante vitrectomía de un paciente diabético con desprendimiento por tracción macular. A: en la fotografía de fondo aparecen exudados duros maculares y pliegues y una contracción focal en la arcada temporal inferior. B: en la OCT se puede ver una banda que tracciona sobre la zona macular. C: aspecto de la macula después de la vitrectomía. Los exudados están en regresión y han desaparecido los pliegues y la contracción focal de la arcada temporal inferior. D: en la OCT se observa la desaparición de la tracción. La mácula ha recuperado su configuración, aunque persiste un discreto acúmulo de líquido intraretiniano. 
retina menos engrosada. Esto aumentaría la eficacia del láser y prolongaría el efecto de la triamcinolona. También los resultados de estos estudios, que en general, han incluido a un escaso número de pacientes, han arrojado datos contradictorios. Así, mientras algunos encuentran beneficios en este tratamiento combinado ${ }^{32-34}$, para otros esta opción no proporciona ventajas significativas $^{35,36}$. De cualquier forma, la Red para la Investigación Clínica de la Retinopatía Diabética esta valorando actualmente la combinación de triamcinolona y láser, la combinación de ranibizumab y láser o el ranibizumab aislado para el edema macular diabético ${ }^{37}$.

Por último y en lo que respecta a la inyección de sustancias antiangiogénicas, tanto el ranibizumab como el pegaptanib podían conseguir a corto plazo la desaparición del edema y no tendrían tantos efectos secundarios como la triamcinolona intravítrea. Aunque los resultados iniciales con estas sustancias parecen alentadores $^{9,10}$ estas buenas perspectivas deberán ser confirmadas en estudios a largo plazo.

Podemos concluir diciendo que mientras estudios prospectivos randomizados con un número suficiente de pacientes y un diseño adecuado no demuestren lo contrario, la fotocoagulación con láser y el control metabólico son los el únicos tratamientos cuya eficacia está claramente demostrada en el edema macular diabético. Cualquier nueva alternativa terapéutica que se quiera introducir deberá ser comparada en ensayos clínicos con la fotocoagulación $^{31}$.

\section{BIBLIOGRAFÍA}

1. Moss SE, KLeIN R, KLeIN BE. The incidence of vision loss in a diabetic population. Ophthalmology 1988; 95: 1340-1348.

2. Kim BY, SMITH SD, KAISER PK. Optical coherence tomographic patterns of diabetic macular edema. Am J Ophthalmol 2006; 142: 405-412.

3. DCCT Research Group. The effect of intensive treatment of diabetes in the development and progression of long-term complications in insulin-dependent diabetes. N Engl J Med 1993: 329: 977-986.

4. UK Prospective Diabetes Study (UKPDS) Group. Intensive blood-glucose control with sulphonylureas or insulin compared with conventional treatment and risk complications in patients with type 2 diabetes (UKPDS 33). Lancet 1998; 317: 703713.

5. The Early Treatment Diabetic Retinopathy Study Research Group. Photocoagulation for diabetic macular edema. Early Treatment Diabetic Retinopathy Study report $\mathrm{n}^{\mathrm{O}}$ 1. Arch Ophthalmol 1985; 103: 1796-1806.

6. JONAS JB, SOFKER A. Intraocular injection of crystalline cortisone as adjunctive treatment of diabetic macular edema. Am J Ophthalmol 2001; 132: 425-427.

7. Martidis A, Duker JS, Greenberg PB, Rogers AH, Puliafito CA, ReICHEL E et al. Intravitreal triamcinolone for refractory diabetic macular edema. Ophthalmology 2002; 109: 920-927.

8. Jonas JB, Kreising I, Sofker K, Degenring RF. Intravitreal injection of triamcinolone for diffuse diabetic macular edema. Arch Ophthalmol 2003; 121: 57-61.

9. Macugen Diabetic Retinopathy Study Group. A phase II randomized double-masked trial of pegaptanib, an antivascular endothelial growth factor aptamer for diabetic macular edema. Ophthalmology 2005; 112: 1747-1757.

10. Chun DW, Heier JS, Topping TM, Duker JS, BANKERT JM. A pilot study of multiple intravitreal injections of ranibizumab in patients with center-involving clinically significant diabetic macular edema. Ophthalmology 2006; 113: 1706-1712

11. Kimura T, Kiryu J, NishiwaKi H, OH H, Suzuma K, WATANABE D et al. Efficacy of surgical removal of the internal limiting membrane in diabetic cystoid macular edema. Retina 2005; 25: 454-461

12. Patel Ji, Hykin PG, Schadt M, LuOng WY, Fitzke F, GREGOR ZJ. Pars plana vitrectomy with and without peeling of the inner limiting membrane for diabetic macular edema. Retina 2006; 26: 5-13.

13. NGUYen QD, SHAH SM, VAN ANDEN E et al. Supplemental inspired oxigen improves diabetic macular edema: a pilot study. Invest Ophthalmol Vis Sci 2003; 45-617-624.

14. DerevjaniK NL, Vinores SA, Xiao W-H, Mori K, Turon T, Hudish $\mathrm{T}$ et al. Quantitative assessment of the integrity of the bloodretinal barrier in mice. Invest Ophthalmol Vis Sci 2002; 43: 2462-2467.

15. BRESNICK GH. Diabetic macular edema. A review. Ophthalmology 1986; 93: 989-997. 
16. WiLKINSON CP, FERRIS FL, KLEIN RE, LEe PP, Agardh CD, Davis $M$ et al. Proposed International Clinical Diabetic Retinopathy and Diabetic Macular Edema Disease Severity Scales. Ophthalmology 2003; 110: 1677-1682.

17. Otani T, Kishi S, Maruyama Y. Patterns of diabetic macular edema with optical coherence tomography. Am J Ophthalmol 1999; 127: 688-693.

18. Pires i, Bernardes RC, Lobo CL, Soares mA, CunHA-VAZ JG. Retinal thickness in eyes with mild nonproliferative retinopathy in patients with type 2 diabetes mellitus: comparison of measurements obtained by retinal thickness analysis and optical coherence tomography. Arch Ophthalmol 2002; 120: 1301-1306.

19. Bandello F, Lanzetta P, Menchini U. When and how to do a grid laser for diabetic macular edema. Doc Ophthalmol 1999; 97: 415-419.

20. Early Treatment Diabetic Retinopathy Study Research Group. Early photocoagulation for diabetic retinopathy. ETDRS report number 9. Ophthalmology 1991; 98: 766-785.

21. LEE CM, OLK RJ. Modified grid laser fotocoagulation for diffuse diabetic macular edema: long term visual results. Ophthalmology 1991; 98: 1594-1602.

22. Early Treatment Diabetic Retinopathy Study Research group. Focal photocoagulation treatment of diabetic macular edema. Relationship of treatment effect to fluorescein angiographic and other retinal characteristics at baseline. ETDRS report $\mathrm{n}^{\circ}$ 19. Arch Ophthalmol 1995; 113: 1144-1155.

23. Lewis H, Abrams GW, Blumenkranz MS, CAmpo RV. Vitrectomy for diabetic macular edema associated with posterior hyaloidal traction. Ophthalmology 1992; 99: 753-759.

24. TACHI N, OGINO N. Vitrectomy for diffuse macular edema in cases of diabetic retinopathy. Am J Ophthalmol 1996; 122: 258-260.

25. Yamamoto $\mathrm{T}$, Akabane $\mathrm{N}$, TAkeuchi $\mathrm{S}$. Vitrectomy for diabetic macular edema: the role of posterior vitreous detachment and epimacular membrane. Am J Ophthalmol 2001; 132: 369-377.

26. Stefaniotou M, Aspiotis M, Kalegeropoulos C et al. Vitrectomy ressults for diffuse diabetic macular edema with and without inner limiting membrane removal. Eur J Ophthalmol 2004; 14: 137-143.

27. Radetzky S, Walter P, Fauser S, Koizumi K, KIRChHOF B, Joussen AM. Visual outcome of patients with macular edema after pars plana vitrectomy and indocyanine green- assisted peeling of the internal limiting membrane. Graefes Arch Clin Exp Ophthalmol 2004; 242: 273-278.

28. Funatsu H, Yamashita H, Sakata K, Noma H, MimuRA T, SuzUKi M et al. Vitreous levels of vascular endotelial growth factor and intercellular adhesión molecule 1 are related to diabetic macular edema. Ophthalmology 2005; 112: 806-816.

29. Inoue M, TaKeda K, Morita K, Yamada M, TANIGAWARA Y, OGUCHI Y. Vitreous concentration of triamcinolone acetonide in human eyes after intravitreal or subtenon injection. Am J Ophthalmol 2004; 138: 1046-1048.

30. Massin P, Audren F, Haouchine B, Erginay A, Bergmann JF, Benosman R et al. Intravitreal triamcinolone acetonide for diabetic diffuse macular edema: Preliminary ressults of a prospective controlled trial. Ophthalmology 2004; 111: 218-225.

31. Diabetic Retinopathy Clinical Research Network. A randomized clinical trial comparing intravitreal Triamcinolonide acetonide and focal/grid photocoagulation for diabetic macular edema. Ophthalmology 2008; 115: 1447-1459.

32. Tunc M, Onder HI, Kaya M. Posterior subTenon's capsule Triamcinolone injection combined with focal laser photocoagulation for diabetic macular edema. Ophthalmology 2005; 112: 1086-1091.

33. Kang SW, SA HS, Chо HY, KIM JI. Macular grid photocoagulation after intravitreal Triamcinolone acetonide for diffuse diabetic macular edema. Arch Ophthalmol 2006; 124 : 653-658.

34. Shimura M, NaKazawa T, Yasuda K, Shiono T, NiSHIDA K. Pretreatment of posterior subtenon injection of Triamcinolone acetonide has beneficial effects for grid pattern photocoagulation against diffuse diabetic macular oedema. Br J Ophthalmol 2007; 91: 449-454.

35. Diabetic Retinopathy Clinical Research Network. Randomized trial of peribulbar Triamcinolonide acetonide with and without focal photocoagulation for mild diabetic macular edema. A pito study. Ophthalmology 2007; 114: 1190-1196.

36. Lam DS, Chan CK, Mohamed S, LaI TY, LeE WY, LIU DT et al. Intravitreal Triamcinolone plus sequential grid laser versus Triamcinolone or laser alone for treating diabetic macular edema. Six-month outcomes. Ophthalmology 2007; 114: 2162-2167.

37. www.drcr.net; fecha de acceso, 12 de junio de 2008. 